\title{
THE REPORTED STUDY HABITS AND TIME-MANAGEMENT TRENDS OF POST-GRADUATE STUDENTS IN ACCOUNTANCY
}

\section{J. P. Fouché}

School of Accounting Sciences

North-West University

Vanderbijlpark, South Africa

e-mail: jaco.fouche@nwu.ac.za

\section{ABSTRACT}

The objective of this article is to analyse the learning environment of honours chartered accountancy students with the specific focus of identifying their learning activities and timemanagement. A survey was conducted at a university in South Africa after initial focus group interviews. The results indicated that the students feel busy, stressed and overwhelmed. Bad study habits, that have a significant negative relationship with performance, include not doing homework, not participating in class, watching too much TV, often looking at a phone, jumping around between activities and procrastination. Good study habits like doing homework, class participating, being able to manage time, being focused and working hard at undergraduate level showed a significant positive correlation with performance. Clearly more can and should be done to support these students with developing more efficient study habits.

Keywords: learning environment; post-graduate accounting students; learner support; timemanagement; accountancy education; study habits

\section{INTRODUCTION}

'Failure is a teacher. It teaches what you ought to learn.' - Lailah Gifty Akita, Beautiful Quotes.

One Tuesday morning a lecturer walked into a fourth year contact session. These senior students did not prepare, were not participating and yet were very busy studying towards a degree aimed at a professional qualification. They were not performing according to their perceived potential. Where did he fail them? More importantly, what could he learn from this?

There is a need for more qualified accountants in South Africa (SAICA 2008, X). Yet, students and academics report various barriers to success. Even the Higher Education Quality Committee (HEQC) has identified the enhancement of teaching and learning as a priority with its Quality Enhancement Project. Student success rates are a great concern to all role players in higher education institutions (De Jager 2014, 54; Gracia and Jenkins 2002, 93). More specifically students often struggle to complete their post-graduate qualifications in 
accountancy (Steenkamp 2012, 2; Sadler and Erasmus 2005, 31). Although there are exceptions, throughput rates at honours and post-graduate level are generally accepted as being less than desired. Major study habits and other factors that were identified as having an effect on the academic success of accounting students include:

- $\quad$ Academic support (De Jager 2014, 56);

- $\quad$ Class attendance (De Jager 2014, 57; Steenkamp 2012, 1);

- $\quad$ Individual commitment and effort (De Jager 2014, 57; Steenkamp 2012, 1);

- $\quad$ Motivation (De Jager 2014, 57);

- $\quad$ Time-management (De Jager 2014, 57; Steenkamp 2012, 1);

- $\quad$ Attending additional help and tutorials (Steenkamp 2012, 1);

- $\quad$ Consistent study (Steenkamp 2012, 1);

- $\quad$ Stress-management (Steenkamp 2012, 1);

- Work-life balance and extracurricular activities (Steenkamp 2012, 1); and

- $\quad$ Study and examination techniques (Steenkamp 2012, 1).

Researchers have reported time as a limited resource (Britton and Tesser 1991, 405). According to Schell $(2014,6)$ students work long hours, sleep too little and do not take sufficient breaks which increases academic stress. Academic stress is also increased as a result of a lack of physical activity (Schell 2014, 4), lack of leisure activities (Misra and McKean 2000, 42), lack of setting goals (Britton and Tesser 1991, 406) and procrastination (Tice and Baumeister 1997, 454). This academic stress has a negative impact on motivation and student performance.

Many authors have already studied the relationship between academic performance, timemanagement, stress as well as various other factors (Misra and McKean 2000; Macan et al. 1990; Ross, Niebling and Heckert 1999). From an interpretivist paradigm it would be important to understand this phenomenon amongst post-graduate students better. Most previous studies focussed on identifying the factors affecting student success but few attempted to obtain an indepth understanding of the factors.

In her study De Jager $(2014,60)$ found that, although few students attribute their success to time-management, bad time-management was ranked as the number one factor for failure. Self-management and time-management are of the pervasive skills set out in the competency framework of the South African Institute of Chartered Accountants (SAICA 2014, 31). However, from own experience, students complain about an unbearable work load. On the other hand, colleagues have reported students not doing homework, not preparing for class and not even attending class. There should be a reason for this anomaly. As researchers have reported time as a limited resource (Britton and Tesser 1991, 405), optimal use of time would be 
important for students. It is with this in mind that the aim of this study was to better understand the issue of time-management for post-graduate chartered accountancy students to enable lecturers to evaluate whether current support and guidance within the learning environment on this matter is sufficient.

The study was done over a period of two years using mixed research methodologies. An exploratory design was used in year one. An exploratory survey under post-graduate chartered accountancy (often referred to as CTA or certificate in the theory of accounting students) students at a university in South Africa was conducted, followed by focus group interviews with these students. Information gathered in this exercise was used to prepare a comprehensive questionnaire, which focused on the learning activities and time-management of CTA students - this questionnaire was used in the following year.

The remainder of this article discusses literature on study habits and time-management of students, the problem statement, research methodology and findings.

\section{STUDY HABBITS AND TIME-MANAGEMENT}

Researchers have examined a wide range of factors as determinants of academic performance. These individual factors can be subdivided into intellective (cognitive) and non-intellective (non-cognitive) factors (Credé and Kuncel 2008). They also concede that the literature in the non-intellective factors are fragmented and in need of clarification. According to Credé and Kuncel (2008) study habits (which would fall under non-intellective factors) refer to 'the degree to which the student engage in regular acts of studying that are characterized by appropriate studying routines (e.g., reviews of material) occurring in an environment that is conductive to studying'. Nonis and Hudson (2010, 229) acknowledge that studies relating the correlation of study time with academic performance have found conflicting results and unfortunately often do not include for the effectiveness of the study time. Time-management of students can therefore not be investigated in isolation from study habits.

Britton and Tesser $(1991,406)$ rightly point out that student success often depends on managing the completion of a mixture of tasks, with various priorities, deadlines, lengths etcetera. It is therefore understandable that time-management is one of the most traditional topics in the field of learning (García-Ros, Pérez-González and Hinojosa 2004, 167). They continue to say that the biggest complaint that students have is that they do not have enough time to carry out their tasks, agreeing with the author's personal experience and feedback during the exploratory study. This is also supported by De Jager $(2014,61)$ who concluded that bad time-management was ranked as the number one factor for failure. 
In the study of De Jager $(2014,64)$ students responded about not being able to manage time, not coping with the work overload, procrastination and keeping their work until the last minute before tests having a negative effect on their studies. Gracia and Jenkins (2002, 98) found in their study that reasons provided by students for failure include a poor memory; lack of adequate revision, lack of class attendance, poor time-management, low motivation levels and a lack of interest in a subject. Macan et al. $(1990,760)$ commented that in trying to read all the content, meeting deadlines and participating in extracurricular activities, students may become overwhelmed with feelings that there is not enough time in the day to attend to their academic work. Macan et al. (1990, 760) also found potentially important relationships between some aspects of time-management and self-reported performance and stress. Ross, Niebling, and Heckert (1999, 312) found that the biggest sources of stress for students were changes in sleeping habits, breaks, changes in eating habits, new responsibilities and an increase in workload. Again, most of these can be referred back to study habits linked to time-management issues. In a study by Sadler and Erasmus (2005, 33) self-discipline, timely and regular examination preparation were also identified by students and lecturers among factors to influence student success, yet again drawing in the effect of time-management.

Linked to time-management is procrastination. Solomon and Rothblum $(1984,505)$ already reported in 1984 a high frequency of procrastination on academic tasks among students. The main reason for procrastination was a fear of failure; the second was evasiveness of the task and laziness. Tice and Baumeister $(1997,455)$ also investigated procrastination and found that students who procrastinated had lower stress levels during the year but reported higher stress levels and lower grades later in the year.

As in the study of Solomon and Rothblum $(1984,505)$ a study of Anthony $(2013,2)$ concluded that one of the main elements of the post-graduate students was a fear of failure. The volume of work, level of complexity and time pressure added to this fear. Therefore, it seems that the issue of time-management is complex and links with many psychological aspects like procrastination and fear. It was found that academically stronger students were more comfortable in managing their time (Anthony 2013, 2). The results of Britton and Tesser (1991, 408) also showed a relationship between time-management attitudes and skills and student performance. It seems that the students who need to manage their time the best - the weaker students - are less prone to do so. The link may lay in the research of Gracia and Jenkins (2002). Gracia and Jenkins (2002, 103) found that, although students would accept responsibility for their failures, they were less willing to take control over their learning and expected someone else to do that. This was also supported by De Jager $(2014,64)$ who found that students blamed 
factors they perceived outside their control to have an impact on their success. It therefore seems that students are not eager to take control over their time-management, yet Misra and McKean (2000, 41) identified effective time-management as a method used by successful students to reduce stress. They confirmed that having a perceived control over time reduces academic stress.

From the studies of Macan et al. (1990) and Britton and Tesser (1991) factors forming components of student time-management are identified as follows:

- $\quad$ Setting goals

- $\quad$ Prioritizing the set goals

- $\quad$ Planning, prioritising and scheduling tasks to achieve the goals and then

- $\quad$ Carrying out the tasks.

According to (Britton and Tesser 1991, 405) students' time is a limited resource and can therefore be more or less effectively managed. It was indeed reported that students with focus (setting goals, sub-goals and tasks) have better time-management and perform better (Britton and Tesser 1991, 408). The best predictor of performance is however not the amount of time, but rather the activities (study habits) carried out while studying (Delucchi, Rohwer and Thomas 1987, 365; García-Ros, Pérez-González and Hinojosa 2004, 169).

Students often complain about a lack of time for their academic activities. This lack of time and sufficient time-management have been linked to failure as indicated earlier. Students do however not want to take control over their time-management, even though research has shown that a few logical steps would help students to focus. It is however not only the amount of time that students have to study that is important - more important is what students do during the time they study.

The researcher has experienced a lack of preparation for class and doing homework assignments, yet students complain that they are very busy and they say they work hard. The primary objective of the study was therefore to understand the learning environment, study habits and world of the post-graduate chartered accountancy students, with specific reference to time-management. This would be done by:

- Understanding the concept of time-management and related study habits, discussed during the literature review;

- $\quad$ Find out how these post-graduate students spend their time;

- $\quad$ Understand what these post-graduate students do when 'studying'; 
- Understand how 'what they do' affect their academic performance; and

- Make recommendations to assist and support students within the learning environment.

\section{METHODS AND INSTRUMENT}

In order to best investigate the personal learning environment and study habits of the postgraduate chartered accountancy students, it is believed that a mixed method of data collection and a descriptive type of reporting would best assist in reaching the objectives of the study. Exploratory designs, as used in this study, begin with a primary qualitative phase after which the findings are validated or otherwise informed by quantitative results. This is also referred to as a sequential exploratory mixed method strategy (Creswell 2013, 207). The study started in year one with a fairly informal questionnaire (to which 173 students responded), prepared from the author's own experience and observations, just asking students whom prepared for class, did the homework assignments and participated in class. Students also had to indicate their time usage and provide reasons for not preparing and doing homework. This questionnaire was followed by four focus group interviews where participants were randomly selected from the class list. The focus groups consisted of between 8 and 10 students. The questions for discussion were focused on gaining insight into their typical day and how they spend their time and the type of activities they do when studying. Setting objectives, dealing with stress and procrastination were also discussed. From this focus group feedback and taking literature into account, a more comprehensive questionnaire was prepared by the author and administered in the next year.

The next year the sample again consisted of the post-graduate chartered accountancy students. The class was smaller and 161 students participated. The students have class for most days of the week from 8:00 to 13:00. Of the students 57.8 per cent were female. The participants consisted of 71.7 per cent white students and 20.8 per cent black students. Most of the students (39.8\%) were living in an own apartment while 31.7 per cent occupied university residences, 21.1 per cent stayed with parents and relatives and the rest made use of students houses and so forth. Only 8.1 per cent of the students were repeat candidates. Of the participants 59.0 per cent indicated that they had a smart phone while 57.5 per cent indicated that they were studying in their home language.

The first questionnaire was analysed using MS Excel. The focus groups were transcribed and analysed making use of AtlasTi. In the next year the support of statistical consultation services at the North-West University was obtained. The data was analysed using SPSS. Ethical clearance was obtained for the study.

As far as could be established this was the first study attempting to analyse the time- 
management and study practices of post-graduate chartered accountancy students at this indepth level.

\section{RESULTS}

The first set of questions was asked to identify the students' general perceptions on study habits and matters supporting study habits that may be linked to time-management. A five point Likert scale ranging from Strongly Disagree (1) to Strongly Agree (5) was used. The results are shown in Table 1.

Table 1: Factors influencing study habits and time-management

\begin{tabular}{|l|c|c|c|c|}
\hline Statement & N & Mean & Std. Dev. & $\begin{array}{c}\text { \% Agree } \\
\text { and } \\
\text { Strongly } \\
\text { Agree }\end{array}$ \\
\hline I often procrastinate (leave everything till the last minute) & 161 & 2.665 & 1.0837 & 22.50 \\
\hline I jump around between learning activities & 160 & 2.663 & 1.0272 & 22.01 \\
\hline I plan each day & 161 & 3.280 & 0.9368 & 43.67 \\
\hline I am able to manage my time & 161 & 3.373 & 0.9207 & 51.90 \\
\hline I am busy & 160 & 4.263 & 0.7647 & 85.35 \\
\hline I am fully focused when studying, doing homework etc. & 160 & 3.800 & 0.7590 & 67.52 \\
\hline I often look at my phone with regards to SMS's, Facebook, & 161 & 3.180 & 0.9610 & 38.99 \\
\hline I witter etc. & 161 & 3.801 & 0.9735 & 55.70 \\
\hline I feel demotivated & 161 & 2.901 & 1.1522 & 26.88 \\
\hline I feel guilty about my studies & 161 & 3.056 & 1.2412 & 38.36 \\
\hline I feel overwhelmed & 161 & 3.950 & 3.2611 & 59.87 \\
\hline Undergraduate prepared me for CTA & 161 & 2.584 & 1.0756 & 18.35 \\
\hline I worked hard at my undergraduate studies & 161 & 3.652 & 0.9891 & 57.23 \\
\hline We have an optimal amount of contact time & 157 & 3.904 & 0.8226 & 22.50 \\
\hline
\end{tabular}

From Table 1 it can be seen that most of the students (85.35\%) agreed that they feel very busy. On the positive side only 22.5 per cent of students felt they do procrastinate, 22.01 per cent jump around learning activities, while most were focused when studying (67.52\%). On the opposite side only 43.67 per cent of students indicated that they plan their day. Many also agreed (38.99\%) that they often look at their phone. Only 22.5 per cent also agreed that they were happy with the amount of contact time. From these opposing findings it is clear that with only 51.9 per cent of students being positive about being able to manage their time the situation is less than optimal. This may have resulted in 55.7 per cent of the students feeling stressed and 59.87 per cent feeling overwhelmed. It was also clear that although they felt that they worked hard at undergraduate studies (57.23\%) it did not really prepare them for the challenging postgraduate studies with only $18.35 \%$ agreeing to it. 
The next questions attempted to identify the students' involvement in the four main subjects (see Table 2).

Table 2: Involvement in subjects (\% of times)

\begin{tabular}{|l|c|c|c|c|}
\hline & $\begin{array}{c}\text { Financial } \\
\text { Accounting }\end{array}$ & Auditing & Tax & $\begin{array}{c}\text { Management } \\
\text { Accounting } \\
\text { and Finance }\end{array}$ \\
\hline Prepare & 34.5 & 19.2 & 26.1 & 26.7 \\
\hline Homework & 65.2 & 56.1 & 55.7 & 66.1 \\
\hline Participate & 88.2 & 83.0 & 86.7 & 86.6 \\
\hline Attend class & 98.1 & 95.9 & 97.0 & 96.4 \\
\hline
\end{tabular}

It is alarming to note that these students at post-graduate level only prepared for contact session between 19.2 per cent (in the case of auditing) to 34.5 per cent (in the case of financial accounting) of the time. Even the level of doing homework assignments (an activity crucial in studying any accountancy qualification) is not satisfying, being between 55.7 per cent for Tax and 66.1 per cent for Management Accounting and Finance. Students also did not all fully participate (almost 10\% of them) in contact session although they mostly attended all sessions.

The students were next required to submit a time sheet of a typical week. The findings are depicted in Table 3. For completeness the finding of the 2014 questionnaire is also supplied, although it did not differ much from 2015.

Table 3: Weekly time sheet (Hours)

\begin{tabular}{|c|c|c|c|c|c|c|}
\hline & $\begin{array}{c}N \\
2015\end{array}$ & $\begin{array}{l}\text { Mean } \\
2015\end{array}$ & $\begin{array}{l}\text { Std. } \\
\text { Dev. } \\
2015\end{array}$ & $\begin{array}{c}N \\
2014\end{array}$ & $\begin{array}{l}\text { Mean } \\
2014\end{array}$ & $\begin{array}{l}\text { Std. } \\
\text { Dev. } \\
2014\end{array}$ \\
\hline Academic time - Financial accounting & 161 & 19.47 & 5.504 & 173 & 19.58 & 5.645 \\
\hline Academic time - Auditing & 161 & 14.09 & 4.601 & 173 & 12.92 & 3.796 \\
\hline Academic time - Tax & 161 & 15.74 & 4.892 & 173 & 13.84 & 3.723 \\
\hline $\begin{array}{l}\text { Academic time - Management Accounting } \\
\text { and Finance }\end{array}$ & 161 & 16.34 & 6.438 & 173 & 13.28 & 3.813 \\
\hline Total academic time & & 65.63 & & & 59.62 & \\
\hline Sleep & 161 & 52.98 & 10.149 & 173 & 52.02 & 9.503 \\
\hline Exercise & 159 & 4.07 & 4.518 & 173 & 4.81 & 5.435 \\
\hline Relax with friends & 159 & 14.03 & 9.165 & 172 & 10.49 & 9.020 \\
\hline Doing chores & 158 & 4.71 & 5.637 & 173 & \multirow[t]{4}{*}{41.08} & \multirow[t]{4}{*}{17.626} \\
\hline Watching TV & 155 & 6.33 & 6.773 & 173 & & \\
\hline Working/Job & 149 & 0.56 & 2.500 & 173 & & \\
\hline Other & 155 & 21.07 & 16.809 & 173 & & \\
\hline TOTAL & 161 & 168.00 & & & 168.00 & \\
\hline
\end{tabular}

From Table 3 it can be seen that students spend more than 60 hours a week on academic matters. If a six day workweek is taken, this is more than 10 hours a day. This confirms students' belief that they are indeed very busy. The least amount of time is spent on Auditing (14.09 hours per 
week). Students spend more time (19.47 hour per week) on Financial Accounting compared to the other modules. Although it was more or less the same in 2014 the times spend on the other modules increased in 2015 due to interventions by the university to stress the importance of regarding the subjects as equally important. Students traditionally spend more time on Financial Accounting. In the past the weighting in the national exams and at the university was skewed toward Financial Accounting. This has however started to change. It seems for Table 3 that students compensated for this change by spending more time on the other subjects without reducing the time spend on Financial Accounting.

On average students get 7.5 hours of sleep a night and spend around 4 hours per week exercising. They do not seem to watch too much TV. As the students are fulltime students, very little time is spent on jobs.

The next questions attempted to identify the activities the students get involved in while not in a contact session (refer to table 4)

Table 4: Outside contact session academic activities (\%)

\begin{tabular}{|l|c|c|c|c|}
\hline & $\begin{array}{c}\text { Financial } \\
\text { Accounting }\end{array}$ & Auditing & Tax & $\begin{array}{c}\text { Management } \\
\text { Accounting and } \\
\text { Finance (MAF) }\end{array}$ \\
\hline Preparation, reading before class & 9.48 & 6.27 & 7.65 & 8.05 \\
\hline Reading after class & 18.76 & 16.29 & 18.54 & 14.06 \\
\hline Making summaries & 12.58 & 14.07 & 14.07 & 10.89 \\
\hline Doing homework, assignments & 28.75 & 32.29 & 25.92 & 37.81 \\
\hline Active continuous studying & 26.87 & 27.20 & 28.22 & 26.14 \\
\hline Other & 3.57 & 3.87 & 5.59 & 3.05 \\
\hline TOTAL & $\mathbf{1 0 0 . 0 0}$ & $\mathbf{1 0 0 . 0 0}$ & $\mathbf{1 0 0 . 0 0}$ & $\mathbf{1 0 0 . 0 0}$ \\
\hline
\end{tabular}

From Table 4 it is evident that the least amount of time is spent on preparation for class - this is relevant to all modules. Reading after class and making summaries consumes a large amount of time. In all modules, except for Tax, most time is spent on doing homework (one quarter to one third).

A principle component analysis was done on the factors influencing study habits as indicated in Table 1. It resulted in four components being identified (see table 5).

Table 5: Factor analysis on factors influencing study habits

\begin{tabular}{|l|c|c|c|c|}
\hline \multirow{2}{*}{} & \multicolumn{4}{|c|}{ Component } \\
\cline { 2 - 5 } & $\mathbf{1}$ & $\mathbf{2}$ & $\mathbf{3}$ & $\mathbf{4}$ \\
\hline I am able to manage my time & -0.699 & -0.401 & & \\
\hline I am fully focused when studying, doing homework etc. & -0.687 & & & -0.405 \\
\hline I am busy & -0.669 & & & \\
\hline I plan each day & -0.484 & & & -0.414 \\
\hline I worked hard at my undergraduate studies & -0.434 & & 0.412 & \\
\hline
\end{tabular}




\begin{tabular}{|l|c|c|c|c|}
\hline \multirow{2}{*}{} & \multicolumn{3}{|c|}{ Component } \\
\cline { 2 - 4 } & $\mathbf{1}$ & $\mathbf{2}$ & $\mathbf{3}$ & $\mathbf{4}$ \\
\hline I feel overwhelmed & & 0.824 & & \\
\hline I feel stressed & & 0.806 & & \\
\hline I feel demotivated & & 0.750 & & 0.435 \\
\hline I feel guilty about my studies & 0.352 & 0.574 & & \\
\hline Undergraduate prepared me for CTA & & & 0.733 & \\
\hline We have an optimal amount of contact time & & & 0.700 & \\
\hline $\begin{array}{l}\text { I often look at my phone with regards to SMS's, Facebook, Twitter } \\
\text { etc. }\end{array}$ & & & & 0.752 \\
\hline I jump around between learning activities & & & & 0.728 \\
\hline I often procrastinate (leave everything till the last minute) & 0.524 & & & 0.626 \\
\hline
\end{tabular}

From Table 5 it can be seen that the following components could be compiled:

Table 6: Summary of component

\begin{tabular}{|l|c|c|c|}
\hline Component & Number of items & Inter-item correlation & Cronbach's Alpha \\
\hline 1. Good study habits & 5 & 0.239 & 0.606 \\
\hline 2. Negative feelings & 4 & 0.435 & 0.747 \\
\hline 3. Undergraduate and contact time & 2 & 0.214 & 0.347 \\
\hline 4. Bad study habits & 3 & 0.342 & 0.611 \\
\hline
\end{tabular}

The total cumulative variance of the four items was 56.76 per cent. It was decided not the analyse item 3 on undergraduate and contact time further. The remainder of items, where the reliability scores were satisfactory, were used to calculate correlations with the March examination scores.

Table 7: Correlations between study habits, time-management and performance

\begin{tabular}{|c|c|c|c|c|c|}
\hline & & $\begin{array}{c}\text { Financial } \\
\text { accounting }\end{array}$ & Auditing & Tax & MAF \\
\hline \multicolumn{6}{|l|}{ FINANCIAL ACCOUNTING } \\
\hline \multirow[t]{3}{*}{ Prepare for financial accounting } & $\begin{array}{l}\text { Correlation } \\
\text { Coefficient }\end{array}$ & $-0.166^{\star}$ & & & \\
\hline & Sig. (2-tailed) & 0.036 & & & \\
\hline & $\mathrm{N}$ & 159 & & & \\
\hline \multirow[t]{3}{*}{ Do financial accounting homework } & $\begin{array}{l}\text { Correlation } \\
\text { Coefficient }\end{array}$ & & $0.193^{*}$ & $0.299^{* \star}$ & $0.203^{*}$ \\
\hline & Sig. (2-tailed) & & 0.015 & 0.000 & 0.011 \\
\hline & $\mathrm{N}$ & & 158 & 158 & 158 \\
\hline \multirow[t]{3}{*}{$\begin{array}{l}\text { Participate in financial accounting } \\
\text { class }\end{array}$} & $\begin{array}{l}\text { Correlation } \\
\text { Coefficient }\end{array}$ & $0.170^{*}$ & $0.205^{\star \star}$ & & \\
\hline & Sig. (2-tailed) & 0.032 & 0.010 & & \\
\hline & $\mathrm{N}$ & 159 & 159 & & \\
\hline \multirow[t]{3}{*}{ Attend financial accounting class } & $\begin{array}{l}\text { Correlation } \\
\text { Coefficient }\end{array}$ & & & & \\
\hline & Sig. (2-tailed) & & & & \\
\hline & $\mathrm{N}$ & & & & \\
\hline \multirow[t]{3}{*}{$\begin{array}{l}\text { Portion of time spend preparing } \\
\text { for Financial Accounting }\end{array}$} & $\begin{array}{l}\text { Correlation } \\
\text { Coefficient }\end{array}$ & $-0.226^{\star *}$ & & & \\
\hline & Sig. (2-tailed) & 0.005 & & & \\
\hline & $\mathrm{N}$ & 155 & & & \\
\hline
\end{tabular}




\begin{tabular}{|c|c|c|c|c|c|}
\hline & & $\begin{array}{c}\text { Financial } \\
\text { accounting }\end{array}$ & Auditing & Tax & MAF \\
\hline \multirow[t]{3}{*}{$\begin{array}{l}\text { Portion of time spend reading } \\
\text { Financial Accounting }\end{array}$} & $\begin{array}{l}\text { Correlation } \\
\text { Coefficient }\end{array}$ & & & $-0.193^{*}$ & $-0.20^{\star}$ \\
\hline & Sig. (2-tailed) & & & 0.016 & 0.013 \\
\hline & $\mathrm{N}$ & & & 154 & 154 \\
\hline \multirow[t]{3}{*}{$\begin{array}{l}\text { Portion of time spend doing } \\
\text { financial Accounting summaries }\end{array}$} & $\begin{array}{l}\text { Correlation } \\
\text { Coefficient }\end{array}$ & & & & \\
\hline & Sig. (2-tailed) & & & & \\
\hline & $\mathrm{N}$ & & & & \\
\hline \multirow[t]{3}{*}{$\begin{array}{l}\text { Portion of time spend doing } \\
\text { Financial Accounting homework }\end{array}$} & $\begin{array}{l}\text { Correlation } \\
\text { Coefficient }\end{array}$ & $0.194^{\star}$ & & $0.189^{*}$ & \\
\hline & Sig. (2-tailed) & 0.016 & & 0.018 & \\
\hline & $\mathrm{N}$ & 155 & & 155 & \\
\hline \multirow[t]{3}{*}{$\begin{array}{l}\text { Portion of time spend actively } \\
\text { studying Financial Accounting }\end{array}$} & $\begin{array}{l}\text { Correlation } \\
\text { Coefficient } \\
\end{array}$ & & & & \\
\hline & Sig. (2-tailed) & & & & \\
\hline & $\mathrm{N}$ & & & & \\
\hline \multirow[t]{3}{*}{$\begin{array}{l}\text { Academic time spend on Financial } \\
\text { Accounting }\end{array}$} & $\begin{array}{l}\text { Correlation } \\
\text { Coefficient }\end{array}$ & $0.176^{*}$ & $0.156^{\star}$ & & \\
\hline & Sig. (2-tailed) & 0.026 & 0.049 & & \\
\hline & $\mathrm{N}$ & 159 & 159 & & \\
\hline \multicolumn{6}{|l|}{ AUDITING } \\
\hline \multirow[t]{3}{*}{ Prepare for auditing class } & $\begin{array}{l}\text { Correlation } \\
\text { Coefficient } \\
\end{array}$ & $-0.170^{*}$ & & & \\
\hline & Sig. (2-tailed) & 0.032 & & & \\
\hline & $\mathrm{N}$ & 159 & & & \\
\hline \multirow[t]{3}{*}{ Do Auditing homework } & $\begin{array}{l}\text { Correlation } \\
\text { Coefficient }\end{array}$ & & $0.224^{\star *}$ & $0.343^{* \star}$ & $.279^{\star \star}$ \\
\hline & Sig. (2-tailed) & & 0.005 & 0.000 & 0.000 \\
\hline & $\mathrm{N}$ & & 158 & 158 & 158 \\
\hline \multirow[t]{3}{*}{ Participate in auditing class } & $\begin{array}{l}\text { Correlation } \\
\text { Coefficient }\end{array}$ & $0.197^{*}$ & $0.273^{\star *}$ & $0.219^{* *}$ & $0.164^{*}$ \\
\hline & Sig. (2-tailed) & 0.013 & 0.000 & 0.006 & 0.039 \\
\hline & $\mathrm{N}$ & 159 & 159 & 159 & 159 \\
\hline \multirow[t]{3}{*}{ Attend auditing class } & $\begin{array}{l}\text { Correlation } \\
\text { Coefficient }\end{array}$ & & $0.192^{*}$ & & \\
\hline & Sig. (2-tailed) & & 0.016 & & \\
\hline & $\mathrm{N}$ & & 159 & & \\
\hline \multirow[t]{3}{*}{$\begin{array}{l}\text { Portion of time spend preparing } \\
\text { for Auditing }\end{array}$} & $\begin{array}{l}\text { Correlation } \\
\text { Coefficient } \\
\end{array}$ & & & & \\
\hline & Sig. (2-tailed) & & & & \\
\hline & $\mathrm{N}$ & & & & \\
\hline \multirow[t]{3}{*}{$\begin{array}{l}\text { Portion of time spend reading } \\
\text { Auditing }\end{array}$} & $\begin{array}{l}\text { Correlation } \\
\text { Coefficient } \\
\end{array}$ & & & & \\
\hline & Sig. (2-tailed) & & & & \\
\hline & $\mathrm{N}$ & & & & \\
\hline \multirow[t]{3}{*}{$\begin{array}{l}\text { Portion of time spend doing } \\
\text { Auditing summaries }\end{array}$} & $\begin{array}{l}\text { Correlation } \\
\text { Coefficient } \\
\end{array}$ & & & & \\
\hline & Sig. (2-tailed) & & & & \\
\hline & $\mathrm{N}$ & & & & \\
\hline \multirow[t]{3}{*}{$\begin{array}{l}\text { Portion of time spend doing } \\
\text { Auditing homework }\end{array}$} & $\begin{array}{l}\text { Correlation } \\
\text { Coefficient }\end{array}$ & $0.187^{\star}$ & $0.176^{*}$ & $0.159^{*}$ & \\
\hline & Sig. (2-tailed) & 0.020 & 0.028 & 0.048 & \\
\hline & $\mathrm{N}$ & 155 & 155 & 155 & \\
\hline \multirow[t]{3}{*}{$\begin{array}{l}\text { Portion of time spend actively } \\
\text { studying Auditing }\end{array}$} & $\begin{array}{l}\text { Correlation } \\
\text { Coefficient } \\
\end{array}$ & & & & \\
\hline & Sig. (2-tailed) & & & & \\
\hline & $\mathrm{N}$ & & & & \\
\hline
\end{tabular}




\begin{tabular}{|c|c|c|c|c|c|}
\hline & & $\begin{array}{c}\text { Financial } \\
\text { accounting }\end{array}$ & Auditing & Tax & MAF \\
\hline \multirow[t]{3}{*}{ Academic time spend on Auditing } & $\begin{array}{l}\text { Correlation } \\
\text { Coefficient }\end{array}$ & & $0.158^{\star}$ & $0.203^{\star}$ & \\
\hline & Sig. (2-tailed) & & 0.047 & 0.010 & \\
\hline & $\mathrm{N}$ & & 159 & 159 & \\
\hline \multicolumn{6}{|l|}{ TAX } \\
\hline \multirow[t]{3}{*}{ Prepare for Tax } & $\begin{array}{l}\text { Correlation } \\
\text { Coefficient }\end{array}$ & $-0.267^{* \star}$ & & & \\
\hline & Sig. (2-tailed) & 0.001 & & & \\
\hline & $\mathrm{N}$ & 159 & & & \\
\hline \multirow[t]{3}{*}{ Do Tax homework } & $\begin{array}{l}\text { Correlation } \\
\text { Coefficient }\end{array}$ & & & $0.275^{\star \star}$ & \\
\hline & Sig. (2-tailed) & & & 0.000 & \\
\hline & $\mathrm{N}$ & & & 158 & \\
\hline \multirow[t]{3}{*}{ Participate in Tax class } & $\begin{array}{l}\text { Correlation } \\
\text { Coefficient }\end{array}$ & $0.185^{\star}$ & $0.218^{\star \star}$ & $0.189^{*}$ & \\
\hline & Sig. (2-tailed) & 0.019 & 0.006 & 0.017 & \\
\hline & $\mathrm{N}$ & 159 & 159 & 159 & \\
\hline \multirow[t]{3}{*}{ Attend Tax class } & $\begin{array}{l}\text { Correlation } \\
\text { Coefficient }\end{array}$ & & $0.179^{*}$ & & \\
\hline & Sig. (2-tailed) & & 0.024 & & \\
\hline & $\mathrm{N}$ & & 159 & & \\
\hline \multirow[t]{3}{*}{$\begin{array}{l}\text { Portion of time spend preparing } \\
\text { for Tax }\end{array}$} & $\begin{array}{l}\text { Correlation } \\
\text { Coefficient }\end{array}$ & $-0.175^{\star}$ & & & \\
\hline & Sig. (2-tailed) & 0.029 & & & \\
\hline & $\mathrm{N}$ & 155 & & & \\
\hline \multirow[t]{3}{*}{ Portion of time spend reading Tax } & $\begin{array}{l}\text { Correlation } \\
\text { Coefficient }\end{array}$ & & & & \\
\hline & Sig. (2-tailed) & & & & \\
\hline & $\mathrm{N}$ & & & & \\
\hline \multirow[t]{3}{*}{$\begin{array}{l}\text { Portion of time spend doing Tax } \\
\text { summaries }\end{array}$} & $\begin{array}{l}\text { Correlation } \\
\text { Coefficient }\end{array}$ & & & & \\
\hline & Sig. (2-tailed) & & & & \\
\hline & $\mathrm{N}$ & & & & \\
\hline \multirow[t]{3}{*}{$\begin{array}{l}\text { Portion of time spend doing Tax } \\
\text { homework }\end{array}$} & $\begin{array}{l}\text { Correlation } \\
\text { Coefficient }\end{array}$ & & & $0.183^{*}$ & $0.184^{*}$ \\
\hline & Sig. (2-tailed) & & & 0.023 & 0.022 \\
\hline & $\mathrm{N}$ & & & 155 & 155 \\
\hline \multirow[t]{3}{*}{$\begin{array}{l}\text { Portion of time spend actively } \\
\text { studying Tax }\end{array}$} & $\begin{array}{l}\text { Correlation } \\
\text { Coefficient }\end{array}$ & & & & \\
\hline & Sig. (2-tailed) & & & & \\
\hline & $\mathrm{N}$ & & & & \\
\hline \multirow[t]{3}{*}{ Academic time spend on Tax } & $\begin{array}{l}\text { Correlation } \\
\text { Coefficient }\end{array}$ & & & & \\
\hline & Sig. (2-tailed) & & & & \\
\hline & $\mathrm{N}$ & & & & \\
\hline \multirow[t]{3}{*}{$\begin{array}{l}\text { Prepare for Management } \\
\text { Accounting and Finance }\end{array}$} & $\begin{array}{l}\text { Correlation } \\
\text { Coefficient }\end{array}$ & & & & \\
\hline & Sig. (2-tailed) & & & & \\
\hline & $\mathrm{N}$ & & & & \\
\hline \multirow[t]{3}{*}{$\begin{array}{l}\text { Do Management Accounting and } \\
\text { Finance homework }\end{array}$} & $\begin{array}{l}\text { Correlation } \\
\text { Coefficient } \\
\end{array}$ & $0.213^{* \star}$ & $0.282^{\star \star}$ & $0.354^{* *}$ & $.289^{* *}$ \\
\hline & Sig. (2-tailed) & 0.007 & 0.000 & 0.000 & 0.000 \\
\hline & $\mathrm{N}$ & 158 & 158 & 158 & 158 \\
\hline \multirow[t]{3}{*}{$\begin{array}{l}\text { Participate in Management } \\
\text { Accounting and Finance class }\end{array}$} & $\begin{array}{l}\text { Correlation } \\
\text { Coefficient }\end{array}$ & $0.184^{\star}$ & & $0.164^{*}$ & $0.159^{*}$ \\
\hline & Sig. (2-tailed) & 0.020 & & 0.039 & 0.046 \\
\hline & $\mathrm{N}$ & 159 & & 159 & 159 \\
\hline
\end{tabular}




\begin{tabular}{|c|c|c|c|c|c|}
\hline & & $\begin{array}{c}\text { Financial } \\
\text { accounting }\end{array}$ & Auditing & Tax & MAF \\
\hline \multirow[t]{3}{*}{$\begin{array}{l}\text { Attend Management Accounting } \\
\text { and Finance class }\end{array}$} & $\begin{array}{l}\text { Correlation } \\
\text { Coefficient }\end{array}$ & & & & \\
\hline & Sig. (2-tailed) & & & & \\
\hline & $\mathrm{N}$ & & & & \\
\hline \multirow{3}{*}{$\begin{array}{l}\text { Portion of time spend preparing } \\
\text { for Management Accounting and } \\
\text { Finance }\end{array}$} & $\begin{array}{l}\text { Correlation } \\
\text { Coefficient }\end{array}$ & $-0.206^{*}$ & & & \\
\hline & Sig. (2-tailed) & 0.010 & & & \\
\hline & $\mathrm{N}$ & 154 & & & \\
\hline \multirow{3}{*}{$\begin{array}{l}\text { Portion of time spend reading } \\
\text { Management Accounting and } \\
\text { Finance }\end{array}$} & $\begin{array}{l}\text { Correlation } \\
\text { Coefficient }\end{array}$ & & & & \\
\hline & Sig. (2-tailed) & & & & \\
\hline & $\mathrm{N}$ & & & & \\
\hline \multirow{3}{*}{$\begin{array}{l}\text { Portion of time spend doing } \\
\text { Management Accounting and } \\
\text { Finance summaries }\end{array}$} & $\begin{array}{l}\text { Correlation } \\
\text { Coefficient } \\
\end{array}$ & & & & \\
\hline & Sig. (2-tailed) & & & & \\
\hline & $\mathrm{N}$ & & & & \\
\hline \multirow{3}{*}{$\begin{array}{l}\text { Portion of time spend doing } \\
\text { Management Accounting and } \\
\text { Finance homework }\end{array}$} & $\begin{array}{l}\text { Correlation } \\
\text { Coefficient }\end{array}$ & $0.253^{* \star}$ & & & $0.202^{\star}$ \\
\hline & Sig. (2-tailed) & 0.002 & & & 0.012 \\
\hline & $\mathrm{N}$ & 155 & & & 155 \\
\hline \multirow{3}{*}{$\begin{array}{l}\text { Portion of time spend actively } \\
\text { studying Management Accounting } \\
\text { and Finance homework }\end{array}$} & $\begin{array}{l}\text { Correlation } \\
\text { Coefficient } \\
\end{array}$ & & & & \\
\hline & Sig. (2-tailed) & & & & \\
\hline & $\mathrm{N}$ & & & & \\
\hline \multirow{3}{*}{$\begin{array}{l}\text { Academic time spend on } \\
\text { Management Accounting and } \\
\text { Finance }\end{array}$} & $\begin{array}{l}\text { Correlation } \\
\text { Coefficient }\end{array}$ & & $0.232^{\star *}$ & $0.242^{\star \star}$ & \\
\hline & Sig. (2-tailed) & & 0.003 & 0.002 & \\
\hline & $\mathrm{N}$ & & 159 & 159 & \\
\hline \multicolumn{6}{|l|}{ NON ACADEMIC TIME } \\
\hline \multirow[t]{3}{*}{ Time spend on sleep } & $\begin{array}{l}\text { Correlation } \\
\text { Coefficient }\end{array}$ & & & & $.234^{\star \star}$ \\
\hline & Sig. (2-tailed) & & & & 0.003 \\
\hline & $\mathrm{N}$ & & & & 159 \\
\hline \multirow[t]{3}{*}{ Time send exercising } & $\begin{array}{l}\text { Correlation } \\
\text { Coefficient } \\
\end{array}$ & & & & \\
\hline & Sig. (2-tailed) & & & & \\
\hline & $\mathrm{N}$ & & & & \\
\hline \multirow[t]{3}{*}{ Time spend relaxing } & $\begin{array}{l}\text { Correlation } \\
\text { Coefficient } \\
\end{array}$ & & & & \\
\hline & Sig. (2-tailed) & & & & \\
\hline & $\mathrm{N}$ & & & & \\
\hline \multirow[t]{3}{*}{ Time spend on chores } & $\begin{array}{l}\text { Correlation } \\
\text { Coefficient } \\
\end{array}$ & & & $-0.190^{\star}$ & \\
\hline & Sig. (2-tailed) & & & 0.017 & \\
\hline & $\mathrm{N}$ & & & 157 & \\
\hline \multirow[t]{3}{*}{ Time spend watching TV } & $\begin{array}{l}\text { Correlation } \\
\text { Coefficient } \\
\end{array}$ & $-0.167^{*}$ & $-0.305^{\star \star}$ & $-0.214^{\star *}$ & \\
\hline & Sig. (2-tailed) & 0.039 & 0.000 & 0.008 & \\
\hline & $\mathrm{N}$ & 153 & 153 & 153 & \\
\hline \multirow[t]{3}{*}{ Time spend on a job } & $\begin{array}{l}\text { Correlation } \\
\text { Coefficient } \\
\end{array}$ & & & & \\
\hline & Sig. (2-tailed) & & & & \\
\hline & $\mathrm{N}$ & & & & \\
\hline \multirow[t]{3}{*}{ Other } & $\begin{array}{l}\text { Correlation } \\
\text { Coefficient } \\
\end{array}$ & & & & \\
\hline & Sig. (2-tailed) & & & & \\
\hline & $\mathrm{N}$ & & & & \\
\hline
\end{tabular}




\begin{tabular}{|c|c|c|c|c|c|}
\hline & & $\begin{array}{c}\text { Financial } \\
\text { accounting }\end{array}$ & Auditing & Tax & MAF \\
\hline \multicolumn{6}{|l|}{ OTHER FACTORS } \\
\hline \multirow[t]{3}{*}{ Bad study habits } & $\begin{array}{l}\text { Correlation } \\
\text { Coefficient }\end{array}$ & & $-0.241^{\star \star}$ & & \\
\hline & Sig. (2-tailed) & & 0.002 & & \\
\hline & $\mathrm{N}$ & & 159 & & \\
\hline \multirow[t]{3}{*}{ Good study habits } & $\begin{array}{l}\text { Correlation } \\
\text { Coefficient }\end{array}$ & $0.258^{* \star}$ & $0.287^{\star \star}$ & $0.307^{\star \star}$ & $0.172^{\star}$ \\
\hline & Sig. (2-tailed) & 0.001 & 0.000 & 0.000 & 0.030 \\
\hline & $\mathrm{N}$ & 159 & 159 & 159 & 159 \\
\hline \multirow[t]{3}{*}{ Negative feelings } & $\begin{array}{l}\text { Correlation } \\
\text { Coefficient }\end{array}$ & & & $-0.263^{\star \star}$ & \\
\hline & Sig. (2-tailed) & & & 0.001 & \\
\hline & $\mathrm{N}$ & & & 159 & \\
\hline
\end{tabular}

${ }^{\star \star}$ Correlation is significant at the 0.01 level (2-tailed)

${ }^{*}$ Correlation is significant at the 0.05 level (2-tailed)

Table 7 only reflects significant correlations. When analysing the study behaviour towards Financial Accounting, preparing for a Financial Accounting class has a significant negative correlation with performance $(\mathrm{P}=0.036)$. The proportion of time spent on preparation also had a significant negative correlation on performance $(\mathrm{P}=-0.005)$. It is interesting to note that doing Financial Accounting homework did only have a significant positive correlation with the other subjects. Participating in the Financial Accounting class had a significant positive correlation $(\mathrm{P}=0.32)$ with performance in Financial Accounting as well as a significant positive correlation $(\mathrm{P}=0.010)$ with Auditing performance. This may be that a large portion of Auditing comprises of investigating the financial measurement and disclosure addressed in Financial Accounting. The proportion of time spend reading Financial Accounting had a significant negative correlation with Tax and Management Accounting and Finance indicating that time spend on Financial Accounting may detract from other subjects. The proportion of time spend doing Financial Accounting homework had a significant positive correlation with performance in Financial Accounting $(\mathrm{P}=0.16)$ and Tax $(\mathrm{P}=0.18)$. Overall academic time spend on Financial Accounting had a significant positive correlation with performance in Accounting $(\mathrm{P}=0.026)$ and Auditing $(\mathrm{P}=0.049)$ corresponding with participating in the Financial Accounting class. Class attendance, doing summaries and actively studying had no significant correlation with performance in Financial Accounting.

Preparing for Auditing had a significant negative correlation with performance in Financial Accounting $(\mathrm{P}=0.005)$. Doing Auditing homework had a significant positive correlation with performance in Auditing $(\mathrm{P}=0.005)$, Tax $(\mathrm{P}=0.000)$ and Management Accounting and Finance $(\mathrm{P}=0.000)$. The proportion of time spent doing Auditing homework also had a significant positive correlation with performance in Auditing $(\mathrm{P}=0.028)$, Financial Accounting $(\mathrm{P}=0.020)$ and $\operatorname{Tax}(\mathrm{P}=0.048)$. Participating in the Auditing class also had a 
significant positive correlation with all subjects. Auditing is one of the subjects that encompass many topics in various subjects - this finding thus makes sense. Attending Auditing Class had a significant positive correlation with performance in Auditing $(\mathrm{P}=0.016)$. The proportion of time spent preparing for Auditing, reading Auditing and doing summaries and actively studying Auditing had no significant correlations with performance in auditing. The total academic time spend on Auditing had a significant positive correlation with performance in Auditing $(\mathrm{P}=0.047)$ and $\operatorname{Tax}(\mathrm{P}=0.10)$.

As with the other subjects mentioned up to now, preparation for Tax classes had a significant negative correlation $(\mathrm{P}=0.001)$ with performance in Financial Accounting. The proportion of time spent on Tax preparation also had a significant negative effect on performance in Financial Accounting $(\mathrm{P}=0.029)$. Doing Tax homework, however, had a significant positive relationship to performance in $\operatorname{Tax}(\mathrm{P}=0.000)$ along with attending Tax classes $(\mathrm{P}=0.024)$. The proportion of time spent on Tax homework also had a significant positive correlation with performance in $\operatorname{Tax}(\mathrm{P}=0.023)$ and Management Accounting and Finance $(\mathrm{P}=0.022)$. Participating in Tax classes had a significant positive correlating with performance in Financial Accounting $(\mathrm{P}=0.019)$, Auditing $(\mathrm{P}=0.006)$ and $\operatorname{Tax}(\mathrm{P}=0.017)$. The proportions of time spend reading, doing summaries, actively studying Tax and the overall academic time spent had no significant correlations with performance.

Doing Management Accounting and Finance homework had a significant positive relationship with performance in all subjects. Participating in Management Accounting and Finance classes also had a significant positive correlation with performance in Management Accounting and Finance $(\mathrm{P}=0.012)$ and Financial Accounting $(\mathrm{P}=0.002)$. Total academic times spend on Management Accounting and Finance had a significant positive relationship with performance in Auditing and Tax. Attending Management Accounting and Finance classes, portion of time spend on reading, portion of time spend doing summaries and portion of time spend actively studying had not significant correlations with performance. The proportion of time spend on preparation did however have a significant negative correlation with performance in Financial Accounting $(\mathrm{P}=0.010)$, like with all other modules.

When analysing study habits it is seen that preparation for class and the proportion of academic time spend on preparation in almost all instances had a significant negative impact on performance in Financial Accounting. Doing homework in one subject on the other hand (with the exception of Tax) had a significant positive relationship with performance in almost all modules. This may be linked to a habit of doing homework. Doing tax homework only significantly affected Tax performance. The amounts of time spend doing homework in 
Financial Accounting and Auditing mostly benefitted performance in all modules.

Class participation in one subject in almost all cases had a significant positive correlation with performance in all other modules. Again this could perhaps be attributed to an attitude and habit of constant hard work. Although class attendance in one module had a positive effect on performance on all modules, the correlation was only significant with the effect of Auditing on Auditing and Tax and the effect of attending Tax classes on Financial Accounting, Auditing and Tax.

The proportion of time spend on reading had in many instances shown a negative correlation with performance in modules, with a significant negative correlation of reading in Accounting on Accounting and reading in Tax on Auditing, Tax and Management Accounting and Finance. This may well indicate that students have ineffective reading skills, which could be taken back to the schooling environment. Making summaries showed a general negative relationship with performance, but none of the correlations were significant. No significant relationships were identified on actively studying. It is sad that the activities the students spend so much time on yielded so little results. The actual total time spend on a module only had a significant positive relationship with regards to Financial Accounting and Auditing.

Time spend on sleep had a significant positive relationship $(\mathrm{P}=0.003)$ on performance in Management Accounting and Finance. This may be because this is probably the module which is less rule driven. Students therefore need to be attentive and being able to think more in analysing and answering questions. Time spend on chores had a significant negative relationship with performance in $\operatorname{Tax}(\mathrm{P}=0.017)$ whereas time spend watching TV had a significant negative effect on Financial Accounting $(\mathrm{P}=0.039)$, Auditing $(\mathrm{p}=.000)$ and Tax $(\mathrm{P}=0.008)$. Times spend on exercising, relaxing and working had no significant effect on performance.

Bad study habits (often looking at a phone, jumping around between activities and procrastinating) had a significant negative correlation with performance in Auditing $(\mathrm{P}=0.002)$, while good study habits (being able to manage time, being focused, working hard at undergraduate level) had a significant positive correlation with all modules ( $\mathrm{P}=0.001,0.000$, 0.000, 0.030 respectively). Having negative feelings (feeling overwhelmed, stressed, demotivated and guilty) had a negative correlation with all subjects with significant negative relationship with performance in $\operatorname{Tax}(\mathrm{P}=0.001)$.

\section{DISCUSSION}

Most of the students feel very busy, feel stressed and overwhelmed with the more than 60 hours 
they spend on average on academic activities per week. Having negative feelings (feeling overwhelmed, stressed, demotivated and guilty) had a negative correlation with all subjects with a significant negative relationship with performance in Tax. This supports previous findings of Macan et al. (1990).

On the positive side, students felt they do not procrastinate or jump around learning activities. They also believed that they were focused when studying. Especially on procrastination this differs from previous findings. Despite thinking they are focused during studying the minority of students indicated that they plan their day and many also agreed that they often look at their phone. From these opposing findings it is clear that with only 51.9 per cent of students being positive about being able to manage their time the situation is less than optimal. It is, to a large extent, alarming to note that students at post-graduate level do not prepare for contact sessions and just more than half of the time do homework assignments. Although they mostly attend the contact sessions they do not all fully participate in class. Time spend watching TV also had a significant negative effect on performance in Financial Accounting, Auditing and Tax. Bad study habits (often looking at a phone, jumping around between activities and procrastinating) had a significant negative correlation with performance in Auditing. It is therefore clear that bad study behaviours would negatively affect performance.

Where students spent more time preparing for Auditing, Tax and Management Accounting and Finance classes it showed in most instances a significant negative correlation with performance in Financial Accounting, indicating a detraction from the focus on Financial Accounting. The proportion of time spend on reading had in many instances showed a negative correlation with performance in modules, with a significant negative correlation of reading in Accounting on Accounting and reading in Tax on Auditing, Tax and Management Accounting and Finance. Making summaries showed a general negative relationship with performance, but none of the correlations were significant. It would be necessary to investigate this seemingly 'good' behaviour's negative effect on performance. It may be linked again to bad timemanagement in not getting the most out of the limited amount of time. It may also indicate that these activities are not the important ones to focus on at post-graduate studies.

Doing homework in one subject on the other hand had a significant positive relationship with performance in all other modules. The amounts of time spend doing homework in Financial Accounting and Auditing mostly benefitted performance in all modules. Class participating in one subject in almost all cases had a significant positive correlation with performance in all other modules. This is also supported by the findings that other good study habits (being able to manage time, being focused and working hard at undergraduate level) had 
a significant positive correlation with all modules.

No significant relationships were identified on the portion of time spend on actively studying. The actual total time spend on a module only had a significant positive relationship with regards to Financial Accounting and Auditing. Times spend on exercising, relaxing and working also had no significant effect on performance.

\section{CONCLUSION AND RECOMMENDATIONS}

The aim of this study was to understand the learning environment and study habits of the postgraduate chartered accountancy students, with specific reference to time-management. Previous studies showed that time-management had an effect on academic performance (De Jager 2014, 64; Macan et al. 1990, 764) and that it is an important area for study to be able to assist students (Ross, Niebling, and Heckert 1999, 312).

The typical students at the selected institution would spend 66 hours on academic related work per week, 53 hours on sleep (7.5 hours per day), 4 hours on exercise, 14 hours on relaxing with friends, 5 hours on chores and 6 hours watching TV. Outside of class (20-25 hours per week), students would spend between 6 per cent to 9 per cent of their time on preparing for class, between 14 per cent and 19 per cent on reading the content after class, 11-14 per cent of their time making summaries, 26-38 per cent of the time doing homework and 26-28 per cent of the time actively studying.

The study found that post-graduate chartered accountancy students at the selected university do feel very busy, stressed and overwhelmed with the more than 60 hours they spend on average on academic activities per week. Although they feel they do not procrastinate and are focused the minority actually plan their day and often interact with their phones. This could well be looking at social network monitoring and exchange/interchanges as many indicated that they had smart phones. The result was that only half the students were positive over managing their time.

Bad study habits that were shown to have a negative significant relationship with performance include not doing homework, not participating in class, watching too much TV, often looking at a phone, jumping around between activities and procrastinating.

Good study habits like doing homework, class participating, being able to manage time, being focused and working hard at undergraduate level showed significant positive correlation with performance.

It is evident that more can and should be done to support students on the soft skill side (including time-management) of their studies. Based on this the department in which this 
programme is offered (as well as similar departments) may wish to consider supplying students with support:

- $\quad$ Regarding time-management

- With regards to bad and good study habits specifically at post-graduate level

Macan et al. (1990, 760) said that the basic recommendations on time-management identified from various sources were:

- $\quad$ to identify needs and wants (objectives and goals)

- $\quad$ prioritise them

- $\quad$ then plan, allocate time and execute.

Macan et al. (1990, 760) found a significant correlation between scores on time-management and attending a seminar on time-management. The finding of this study identify a gap in the time-management and related activities of students and the findings can therefore be used to design a presentation or seminar for post-graduate chartered accountancy students on timemanagement as well as on bad and good study habits. It also shows the importance of supplying students with emotional support.

This study adds to the literature on time-management. It provides a unique contribution to the time-management practices of specifically post-graduate charted accountancy students. It also supports the finding of Delucchi, Rohwer and Thomas $(1987,365)$ and (García-Ros, PérezGonzález, and Hinojosa 2004, 169) that the best predictor of performance is however not the amount of time, but rather the activities carried out while studying. The finding help academics and students to better understand the learning environment of the CTA students. The findings of the study will also assist universities in planning and implementing efficient and effective learning support. Improved knowledge and better support systems should contribute to improved throughput, and therefore addressing the accounting skills shortage in South Africa.

\section{LIMITATIONS AND FURTHER RESEACH}

A limitation of the study was the fact that it was only done at a single institution. As this was an exploratory study this is acceptable. Although the results may not be that generalisable, what the students experience may well be found at any of the other 14 SAICA accredited universities as the broader programme structure and competency frameworks on which it is based are more or less the same. The link between the low value of reading and preparation and learner 
academic literacy and reading skills can also be investigated.

Further research is needed to elaborate and follow-up on the findings of this study. Especially on why preparing for class and making summaries do not positively correlate to academic performance. This study also needs to be done at other institutions to increase the generalisation of it.

\section{ACKNOWLEDGEMENT}

A special word of thanks to my colleague, Desmond Phuthi, for assisting with the focus group interviews. Thank you also to Dr Suria Ellis for the statistical analysis.

\section{REFERENCES}

Anthony, James. 2013. An exploratory investigation of the approaches to learning of accounting students studying toward a professionally accredited post-graduate programme at the University of Cape Town. https://open.uct.ac.za/handle/11427/6588 (accessed 12 November 2015).

Britton, Bruce K. and Abraham Tesser. 1991. Effects of time-management practices on college grades. Journal of Educational Psychology 83(3): 405-410.

Credé, Marcus and Nathan R. Kuncel. 2008. Study habits, skills, and attitudes: The third pillar supporting collegiate academic performance. Perspectives on Psychological Science 3(6): 425453.

Creswell, John W. 2013. Research design: Qualitative, quantitative, and mixed methods approaches. Sage publications.

De Jager, Eloise. 2014. Thuthuka students' perceptions of factors influencing success. Journal of Economic and Financial Sciences 7(1): 53-72.

Delucchi, Joanne Jensen, William D. Rohwer and John W. Thomas. 1987. Study time allocation as a function of grade level and course characteristics. Contemporary Educational Psychology 12(4): 365-380.

García-Ros, Rafael, Francisco Pérez-González and Eugenia Hinojosa. 2004. Assessing time management skills as an important aspect of student learning the construction and evaluation of a time management scale with Spanish high school students. School Psychology International 25(2): 167-183.

Gracia, Louise and Ellis Jenkins. 2002. An exploration of student failure on an undergraduate accounting programme of study. Accounting Education 11(1): 93-107.

Macan, Therese H., Comila Shahani, Robert L. Dipboye and Amanda P. Phillips. 1990. College students' time management: Correlations with academic performance and stress. Journal of Educational Psychology 82(4): 760.

Misra, Ranjita and Michelle McKean. 2000. College students' academic stress and its relation to their anxiety, time management, and leisure satisfaction. American Journal of Health Studies 16(1): $41-51$

Nonis, Sarath A. and Gail I. Hudson. 2010. Performance of college students: Impact of study time and study habits. Journal of Education for Business 85(4): 229-238.

Ross, Shannon E., Bradley C. Niebling and Teresa M. Heckert. 1999. Sources of stress among college students. College Student Journal 33(2): 312.

Sadler, E., and B. J. Erasmus. 2005. The academic success and failure of black chartered accounting graduates in South Africa: A distance education perspective. Meditari Accountancy Research 
13(1): 29-50.

SAICA. 2008. Skill Shortage Report. Johannesburg: SAICA.

SAICA. 2014. Competency framework detailed guidance for academic programmes: Competencies of a CA(SA) at the point of the Initial Test of Competence (ITC) (assessment of core technical knowledge). Johannesburg: SAICA.

Schell, P. 2014. Work less, do more, live better. http://www.patheos.com/blogs/markdroberts/ 2014/09/09/work-less-do-more-live-better/ (accessed 12 November 2015).

Solomon, Laura J. and Esther D. Rothblum. 1984. Academic procrastination: Frequency and cognitivebehavioral correlates. Journal of Counseling Psychology 31(4): 503-509.

Steenkamp, G. 2012. The impact of study behaviour on the success of South African CTA students. SA Journal of Accounting Research 26(1).

Tice, Dianne M. and Roy F. Baumeister. 1997. Longitudinal study of procrastination, performance, stress, and health: The costs and benefits of dawdling. Psychological Science: 454-458. 